\title{
Superconductivity in compressed iron: Role of spin fluctuations
}

\author{
I.I. Mazin, D.A. Papaconstantopoulos, and M.J. Mehl \\ Center for Computational Materials Science, \\ Naval Research Laboratory, Washington, DC 20375-5000, USA
}

\begin{abstract}
The recent discovery of superconductivity in hexagonal iron under pressure poses a question about whether it is of conventional (phonon) or unconventional (magnetic?) origin. We present first-principles calculations of the electron-phonon coupling in iron at $P \gtrsim 15 \mathrm{GPa}$, and argue that a conventional mechanism can explain the appearance of superconductivity, but not its rapid disappearance at $P \gtrsim 30 \mathrm{GPa}$. We suggest that spin fluctuations, ferro- and/or antiferromagnetic, play a crucial role in superconductivity in this case.
\end{abstract}

74.70.Ad, 78.20.Bh, 72.20.Dp

The recent report of superconductivity in Fe under pressure 1 was among the latest news in the chain of discoveries of interesting cases of superconductiyity in conventional materials: $\mathrm{MgB}_{2}$ a, $\mathrm{MgCNi}_{3}$ 目, $\mathrm{ZrZn}_{2}$ 过, and others. At first glance, there is nothing unexpected in the fact that iron, a transition metal, becomes superconducting in a nonmagnetic phase. However, a closer look at this case uncovers a number of strange facts which do not square with this simplistic scenario. First, in the phase diagram (Fig.1) according to Ref. 1 the superconductivity appears at the same pressure $(P \approx 15 \mathrm{GPa})$, at which the bcc-hcp phase transition takes place. In other words, the bcc phase is not superconducting (and is magnetic), while the hcp phase is already superconducting at the lowest pressure at which it is stable. What is surprising, however, is that the critical temperature is zero at the phase transition pressure, and then grows gradually with pressure. If the superconductivity were due to phonons, its appearence exactly at the crystallographic phase transition would be just a coincidence, since there is no reason for superconductivity to be suppressed near such a (first order) phase transition. Second, the superconductivity disappears very rapidly with increasing pressure. Essentially, superconductivity occurs only in a narrow range of compression, $132 \mathrm{Bohr}^{3}<V<145 \mathrm{Bohr}^{3}$, where $V$ is the unit cell volume (with two atoms per cell). As we show below, neither electronic (e.g., density of states) characteristics nor phonon frequencies change with pressure rapidly enough to reduce the critical temperature from nearly $2 \mathrm{~K}$ to zero at such a small relative compression.

On the other hand, there are cases in nature when superconductivity exists only in a narrow pressure range, as in the recently discovered ferromagnetic superconductor $\mathrm{UGe}_{2} .5$ The accepted explanation for such behavior in this case is that superconductivity there is believed to be induced by spin fluctuations, which, first, stiffen rapidly away from the quantum critical point (that is, the pressure that corresponds to the Curie temperature $T_{C}=0$ ), and, second, grow in magnitude close to this point. Thus, the critical temperature goes to zero at the critical point, then rises away from it, but disappears again when the energy of the spin fluctuations becomes too high.6.

Finally, it is not even clear whether hcp iron is in- deed nonmagnetic over its whole range of stability. Density functional calculations using the Generalized Gradient Approximation (GGA), which is usually very reliable for $3 \mathrm{~d}$ metals, clearly show an antiferromagnetic ground state 8 , or a noncollinear magnetic state 9 , but not a paramagnetic one. Furthermore, the calculated elastic properties in magnetic hcp iron are in good agreement with experiment, while those for a nonmagnetic iron seriously disagree with experiment 8 Admittedly, there is one experiment that is hard to explain if hcp iron is magnetic even locally, namely Mössbauer spectroscopy under pressure 10. Given this contradiction, we take the point of view that the question of magnetism in hcp iron is still open, and even if the hcp iron is in fact non-magnetic, it should be very close to a magnetic instability.

In 1979, well before superconductivity was discovered in hcp iron, Wohfarth pointed out that spin-fluctuations may play a role.11 He noticed that at the lowest pressures at which the hcp iron is stable, it must be very close to an antiferromagnetic instability, which should lead to pair-breaking spin fluctuations. He also pointed out that these fluctuations should disappear at higher pressure, as the density of states becomes smaller, and thus at some pressure superconductivity is to be expected. Of course, what is missing from this scenario is the fact that superconductivity disappears again rather soon when pressure is increased.

In order to elucidate the potential for conventional, electron-phonon superconductivity in iron we performed first-principles Linear Augmented Plane Wave (LAPW) calculations in GGA of the following quantities: (a) the electron-ion matrix element, $W$, (b) the density of states at the Fermi level, $N$, and (c) the Stoner factor, $I$. Superconductivity in transition metals has been subject to numerous studies in the 70's and 80's (see, e.g., Ref. 12 for review), and is quantitatively understood. The McMillan formula,

$$
T_{c}=\frac{\omega_{\log }}{1.2} \exp \left[\frac{-1.02(1+\lambda)}{\lambda-\mu^{*}(1+0.54 \lambda)}\right],
$$

describes $T_{c}$ reasonably well. The parameters of the formula have the following meanings: $\omega_{\log }$ is the logarithmically averaged phonon frequency, $\mu^{*}$ is the Coulomb 
pseudopotential, which for transition metals is usually close to 0.1 , and $\lambda$ is the electron-phonon coupling constant, $\lambda=\eta M^{-1}\left\langle\omega^{-2}\right\rangle$, where $\eta=N W$ is the so-called Hopfield parameter, $M$ is the ion mass, and $\left\langle\omega^{-2}\right\rangle$ is the average inverse square of the phonon frequency. Note that the electron-phonon coupling is responsible for both pairing interaction and mass renormalization. The constant $\lambda$ measures both effects and appears three times in the McMillan formula: once as a measure of the pairing strength, and twice (in $1+\lambda$ and in $1+0.54 \lambda$ ) because of mass renormalization.

In at least one transition metal, $\mathrm{Pd}$, the electronphonon coupling is definitely strong enough to render the material superconducting but, because it is near a magnetic instability, superconductivity is suppressed. In other words, charge fluctuations (phonons) coexist in $\mathrm{Pd}$ with spin-fluctuations (paramagnons), which have a pairbreaking effect for s-wave symmetry, but contribute to the mass renormalization like phonons. These effects can be approximately accounted for by modifying the McMillan formula as follows:

$$
T_{c}=\frac{\omega_{\log }}{1.2} \exp \left\{\frac{-1.02\left(1+\lambda_{p h}+\lambda_{s f}\right)}{\lambda_{p h}-\lambda_{s f}-\mu^{*}\left[1+0.54\left(\lambda_{p h}+\lambda_{s f}\right)\right]}\right\},
$$

where the subscripts stand for "phonons" and "spin fluctuations", so that $\lambda_{s f}$ is an electron-paramagnon coupling constant.

Since the Migdal theorem does not hold for paramagnons, it is hardly possible to derive a formula for $\lambda_{s f}$ and compute it from first principles. Within the framework of the Stoner model, and near the magnetic instability, one can sstimate coupling with ferromagnetic spin fluctuations as

$$
\lambda_{s f} \propto\left\langle\frac{1}{1-N I}\right\rangle \approx \frac{\alpha}{1-N I},
$$

where $I$ is the Stoner factor and $\alpha$ characterizes the band structure and the $q$-dependence of the bare magnetic susceptibility 13 . The most accurate way to estimate $I$ is by performing fixed spin moment calculations for small moments and taking the second derivative of the total energy with respect to the fixed moment, $I=N^{-1}-2 d^{2} E / d m^{2}$. With this method we calculated $I=0.075 \mathrm{Ry} /$ atom, using fixed-spin-moment LAPW total energies at $V=146.53 \mathrm{Bohr}^{3}$. Note that $I$ is practically pressure-independent.

For antiferromagnetic spin fluctuations Eq 3 is not applicable, but instead one can write, as a first approximation,

$$
\lambda_{s f} \propto\left\langle\frac{1}{1-\chi I}\right\rangle \approx \frac{\alpha}{1-\chi I},
$$

where $\chi$ is the noninteracting spin susceptibility at the wave vector corresponding to the antiferromagnetic ordering. The main effect of pressure on the electronic structure of hcp iron is an overall scaling of the bandwidth. Thus, one can assume that $\chi \approx \beta N$, where $\beta$ is a pressure independent constant.

All quantities entering Eq. 2, except $\alpha$ and $\beta$, were calculated from first principles, using the approximations outlined ahove with the phonon frequencies, calculated elsewhere 14, which agree well with experiment15. It is worth noting that because of all these approximations we do not expect to obtain quantitatively the value for $T_{c}$, but we do expect to describe correctly the trends associated with the pressure 16 .

In the calculations of the electron-phonon Hopfield parameter $\eta$, we used the "rigid-muffin-tin" theory of Gaspari and Gyorffy 17 According to this theory, which is known to work quite well for transition metals (see, e.g. Ref. 18), $\eta=\sum_{l} W_{l}^{2} N_{l} N_{l+1} / N$, where $W_{l}$ are computed from particular integrals involving the radial wave functions, as defined in Ref. 17, and $N_{l}$ is the partial density of states at the Fermi level with angular momentum $l$. We used self-consistent MT potentials with touching MT spheres, calculated by the LAPW method. It is well known 18 that for transition metals the main contribution to $\eta$ comes from $l=2$, thus $\eta \approx W_{d}^{2} N_{d} N_{f} / N \approx W_{d}^{2} N_{f}$, as the ratio $N_{d} / N$ is close to unity in transition metals. In this connection, it is worth noting that the volume dependence of $\eta$ (cf. Fig.2) is totally different from that of the total density of states $N$; in fact it has the opposite variation.

The pressure-independent constants $\alpha$ and $\beta$ were adjusted so as to have the magnetic instability $(\beta N I=1)$ near the hcp-bcc phase boundary, $V \approx 145 \mathrm{Bohr}^{3}$, and to have the maximal critical temperature of the right order. We used $\alpha=0.13$ and $\beta=1.215$. Qualitative dependence of $T_{c}$ on pressure is not sensitive to the actual values of these constants.

The results of our calculations are summarized in Table 1, and compared with experiment on Fig.1. We observe that the phase diagram can be described qualitatively within the framework of the conventional scenario (pairing phonons plus pair-breaking spin fluctuations), in the sense that the critical temperature rises with pressure near the bcc-fcc transition pressure, reaches a maximum, and then decays. The underlying physics is as follows: spin fluctuations, which get stronger with lowering pressure, suppress superconductivity, and at very high pressures the lattice stiffens and $T_{c}$ goes down. However, the range of pressures at which superconductivity exists is much larger in the calculations. This is not accidental; the only mechanism that prevents superconductivity at higher pressure is stiffening of the lattice, since the electronic part, $\eta$, actually grows with pressure (Fig.2). Indeed, hcp Fe does stiffen rapidly under pressure. However, it is by far not strong enough an effect to destabilize superconductivity at $P>35 \mathrm{GPa}$ (corresponding to the atomic volume of $132 \mathrm{Bohr}^{3}$, which is only a $4 \%$ compression from the maximum $T_{c}$ volume). In other words, elastic properties do not change fast enough with pressure to explain such an extraordinarily narrow range of 
superconductivity.

On the other hand, magnetic properties, at least as calculated within density functional theory, do change in the relevant range. Indeed, at $V=180 \mathrm{Bohr}^{3}$, both antiferromagnetic arrangements considered in Ref. \& are stable and produce the same magnetic moment, on the order of $2 \mu_{B}$ per Fe atom 19 . At $V=145 \mathrm{Bohr}^{3}$, the largest volume at which hcp iron exists, both arrangements are still stable, and the corresponding magnetic moments are similar, although they reduce to values of $\approx 0.5 \mu_{B}$. One can interpret this as local magnetism on $\mathrm{Fe}$ with nearestneighbor antiferromagnetic exchange $(J)$. The exchange energy for the magnetic structure afmI (we use the notation of Ref. 8) is zero, for afmII it is $-2 J$ per atom, and for a ferromagnetic state it is $+6 J$ per atom. Apparently, the latter energy is sufficiently large to render the ferromagnetic state unstable. In fact, and this has not been noticed before, while the ferromagnetic state is energetically unfavorable compared with the nonmagnetic state for all volumes up to $V=157 \mathrm{Bohr}^{3}$, there is a metastable ferromagnetic state (metamagnetism) with $M \approx 2.6 \mu_{B}$ /atom, for all $V>145 \mathrm{Bohr}^{3}$ (Fig.3) On the other hand, while antiferromagnetic states, stabilized by the exchange energy, are lower in energy than the nonmagnetic one, the equilibrium magnetization is rather small, on the order of $1 \mu_{B}$. We find, in agreement with Ref. 8, that the antiferromagnetism disappears only at $V \approx 120 \mathrm{Bohr}^{3}$.

While the picture derived from these calculations is at odds with that deduced from the Mössbauer experiment, in the sense that the calculated tendency to magnetism appears to be too strong, the main conclusion can be expressed qualitatively as follows: the spectrum of the spin fluctuations changes with pressure rapidly and drastically near $P \approx 20 \mathrm{GPa}$, and probably in a very nontrivial way, reflecting two different types of magnetic excitations: antiferromagnetic, and also ferromagnetic (the latter, possibly, of highly nonlinear metamagnetic character). This strongly suggests an exclusive role played by spin fluctuations in iron superconductivity around $P \approx 20 \mathrm{GPa}$. Whether the superconductivity is s-wave, induced by the electron-phonon interaction, with spin fluctuations being ordinary pair breakers, or it is d-wave or p-wave, with spin fluctuations being the pairing agents, cannot be firmly established now, and should be clarified by further experimental studies. However, the latter scenario seems to be not only more exciting, but also more likely, if we believe that, according to the Mössbauer results, there are no localized magnetic moments in the whole superconductivity range, because this means that the spin fluctuations become weaker away from the bcc-hcp transition, and their pair-breaking effect can only diminish with pressure. On the other hand, if superconductivity is d- or p-wave, and the spin fluctuations are pairing, the phase diagram looks fonsistent with the standard prediction for this scenariol. A key experiment in this situation would be measuring the effect of nonmagnetic impurities on superconductivity.
This work was supported by the Office of Naval Research. The authors are grateful to D.J. Singh, A. F. Goncharov and R.E. Cohen for useful discussions.

${ }^{1}$ K. Shimizu, T. Kimura, S. Furomoto, K. Takeda, K. Kontani, Y. Onuki, and K. Amaya, Nature (London), 412, 3169 (2001)

${ }^{2}$ J. Nagamatsu, N. Nakagawa, T. Muranaka, Y. Zenitani and J. Akimitsu, Nature (London) 410, 63 (2001).

${ }^{3}$ T. He, Q. Huang, A.P. Ramirez, Y. Wang, K.A. Regan, N. Rogado, M.A. Hayward, M. K. Haas, J.S. Slusky, K. Inumaru, H.W. Zandbergen, N.P. Ong, and R.J. Cava, condmat/0103296.

${ }^{4}$ C. Peiderer, M. Uhlarz, S.M. Hayden, R. Vollmer, H.v.Lohneysen, N.R. Bernhoeft and C.G. Lonzarich, Nature 412, 58 (2001).

${ }^{5}$ S. S. Saxena et al., Nature (London) 406, 587 (2000).

${ }^{6}$ D. Fay and J. Appel, Phys. Rev. B 22, 3173 (1980).

7 T.R. Kirckpatrick, D. Belitz, T. Vojta, and R. Narayanan, cond-mat/0105627 (2001).

${ }^{8}$ G. Steinle-Neumann, L. Stixrude, and R.E. Cohen, Phys. Rev. B 60, 791 (1999).

${ }^{9}$ R.E. Cohen, S. Gramsch, S. Mukherjee, G. SteinleNeumann, L. Stixrude, cond-mat/0110025.

${ }^{10}$ R. D. Taylor, M. P. Pasternak, and R. Jeanloz, J. Appl. Phys. 69, 6126 (1991).

${ }^{11}$ E.P. Wohlfarth, Phys. Lett. 75A, 141 (1979).

12 D. Rainer, Principles of ab initio calculations of superconducting transition temperatures, in Progress in Low Temperature Physics, Vol. X, p. 373 (North Holland, 1986).

13 The density functional calculations suggest that the spin fluctuations should have substantial $q$-dependence with a maximum away from the zone center. This follows from the fact 8 , that while ferromagnetic ground state is unstable for the whole pressure range of interest, various antiferromagnetic states are stable in the calculations.

${ }^{14}$ D. Alfe, G.D. Price, and M.J. Gillan, Phys. Rev. B 64, 045123 (2001); P. Söderlind, J.A. Moriarty, and J.M. Wills, Phys. Rev. B 51, 14063 (1996).

${ }^{15}$ H. Olijnyk, A.P.Jephcoat, and K. Refson, Europhys. Lett. 53, 504 (2001);

${ }^{16}$ All published calculations and experiments give roughly the same volume dependence for individual phonon modes and for average phonon frequencies. Generally speaking, one may think about a phonon mode, which becomes soft at the bcc-hcp phase transition, and is responsible for superconductivity. This could, in principle, explain the narrow range of the existence of the superconducting phase. However, the calculated dispersion curves (Ref. 14) do not show any unusual mode softening. We also explicitely checked the most likely candidate for a soft mode, the in-plane optical phonon, by performing LAPW frozen phonon calculations, and found that it is not particularly soft at the hcp-bcc $\left(273 \mathrm{~cm}^{-1}\right)$ and hardens with pressure with a normal Grüneisen parameter of 1 (at $V=130 \mathrm{Bohr}^{3}$ ) to 1.7 
(at $V=146 \mathrm{Bohr}^{3}$ ).

17 G.D. Gaspari and B.L. Gyorffy Phys. Rev. Lett. 28, 801 (1972).

18 D.A. Papaconstantopoulos, L.L. Boyer, B.M. Klein, A.R. Williams, V.L. Moruzzi, J.F. Janak, Phys. Rev. B 15, 4221 (1977).

${ }^{19}$ Note that in the Ref. 8 the moments were plotted incorrectly smaller by a factor of two 2 .

${ }^{20}$ A. Jephcoat, H. K. Mao, and P. Bell, J. Geophys. Res. 91, 4677 (1986); H. K. Mao, J. Xu, V. V. Struzhkin, J. Shu, R. J. Hemley, W. Sturhahn, M. Y. Hu, E. E. Alp, L. Vocadlo, D. Alfe, G. D. Price, M. J. Gillan, M. Schwoerer-Böhning, D. Häusermann, P. Eng, G. Shen, H. Giefers, R. Lbbers, G. Wortmann, Science, 292, 914 (2001).

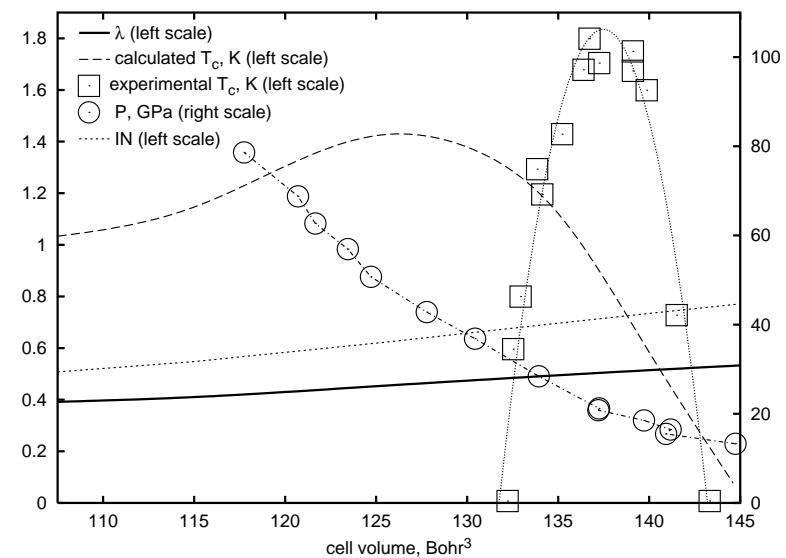

FIG. 1. Volume dependence of experimental and calculated superconducting properties of hcp iron. The electron-phonon coupling constant, $\lambda$, and the critical temperature for the s-wave superconductivity, $T_{c}$, (according to Eq.2) were computed as described in the text. The constant $\alpha$, controlling the strength of the pair-breaking effect of spin fluctuations, was adjusted so as to have superconductivity disappear at $V \approx 145 \mathrm{Bohr}^{3}$, as in the experiment. The experimental critical temperatures from Ref. 1 are also plotted. For translating the pressure scale into the yolume scale we used the experimental equation of state 20, which is also shown in the Figure. We also plotted the Stoner product, $I N$.

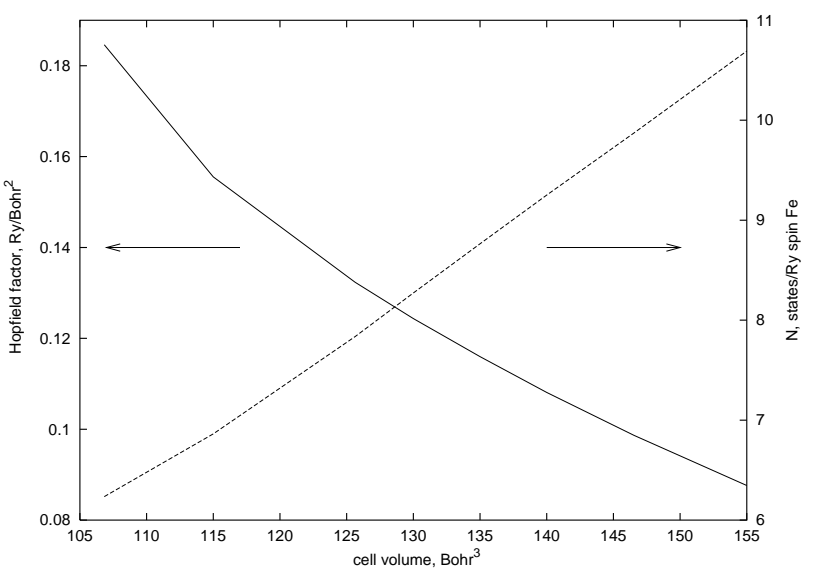

FIG. 2. Hopfield factor $\eta$ in Ry/Bohr ${ }^{2}$ compared with the density of states at the Fermi level, $N$.

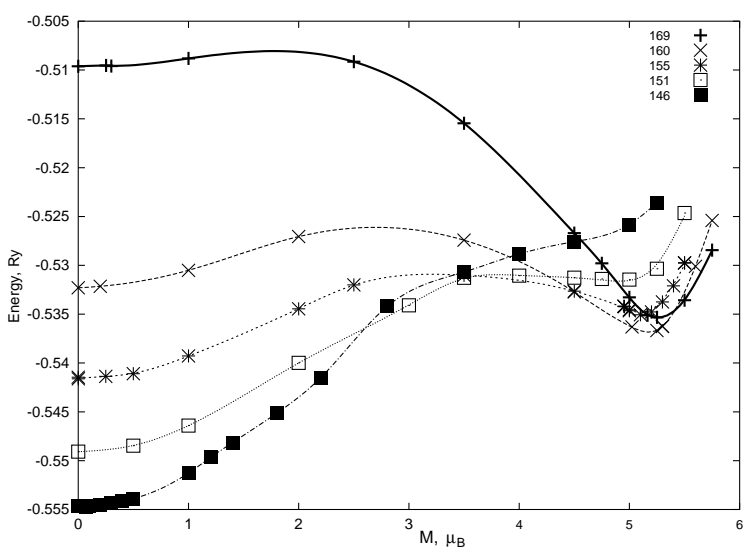

FIG. 3. LAPW fixed spin moment calculations for ferromagnetic hcp iron. Magnetic moments and energies are given on a per cell (two atoms) basis. The curves correspond, from up down, to the volumes per atom: 169.97 Bohr $^{3}$, 160.44 Bohr ${ }^{3}$, 155.81 Bohr ${ }^{3}$, 151.27 Bohr ${ }^{3}$, and $146.83 \mathrm{Bohr}^{3}$.

TABLE I. Calculated parameters at three representative volumes.

\begin{tabular}{llll}
\hline \hline$V, \mathrm{Bohr}^{3}$ & 106.83 & 125.63 & 146.53 \\
$P, \mathrm{GPa}^{(a)}$ & 79 & 48 & 12 \\
$\eta, \mathrm{Ry} / \mathrm{Bohr}^{2}$ & 0.185 & 0.132 & 0.099 \\
$1 / \sqrt{\left\langle\omega^{-2}\right\rangle}, \mathrm{cm}^{-1(b)}$ & 332 & 260 & 206 \\
$\omega_{\text {log }}, \mathrm{cm}^{-1(b)}$ & 343 & 260 & 200 \\
$N, \mathrm{st} . / \mathrm{Ry}$ atom spin & 6.7 & 8.3 & 10.4 \\
$1 /(1-I N)$ & 2.0 & 2.7 & 4.6 \\
$T_{c}, \mathrm{~K}, \mathrm{Eq} 11$ & 2.5 & 4.1 & 6.0 \\
$T_{c}, \mathrm{~K}, \mathrm{Eq} 2(\alpha=0.013, \beta=1.215)$ & 1.0 & 1.4 & 0.0 \\
\hline \hline
\end{tabular}

(a) Ref. 20

(b) These values are calculated from the data in Ref. 14, assuming that the phonon density of states is uniformly distributed between the longitudinal and the transverse peaks. In fact, any reasonable assumption about the shape of the phonon DOS leads to very similar results. 